\title{
Plasma Kinetic Resection of Prostate Combined Terazosin for Treatment of BPH Patient with Coexisting Hypertension
}

\author{
Ming Zheng', Mingqiang Zeng1, Chonghe Jiang2* ${ }^{2}$, Jinyang Chen ${ }^{1}$, Shuren Li ${ }^{1}$, Jiaxin Wang1 \\ ${ }^{1}$ Department of Urology, Xiangtan Hospital of Nanhua University, Xiangtan, China \\ ${ }^{2}$ Department of Urology, Qingyuan City People's Hospital of Jinan University, Qingyuan, China \\ Email: ${ }^{*}$ chinghe.jiang@gmail.com
}

Received 21 April 2014; revised 15 May 2014; accepted 22 May 2014

Copyright (C) 2014 by authors and Scientific Research Publishing Inc.

This work is licensed under the Creative Commons Attribution International License (CC BY). http://creativecommons.org/licenses/by/4.0/

(c) (i) Open Access

\section{Abstract}

Objective: To evaluate the efficacy and safety of terazosin and plasma kinetic resection of the prostate (PKRP) in the treatment of benign prostatic hyperplasia (BPH) patients with coexisting hypertension. Methods: A total of $\mathbf{2 0 5}$ men who suffered from BPH with concomitant hypertension were enrolled in this study. They all received PKRP surgery and terazosin medication from one week before to 3 months after the operation. The procedures of efficacy and safety were evaluated by analysis of the changes in blood pressures, IPSS, Qmax and the drug related adverse events 4 weeks and 3 months after the operation. Results: The systolic blood pressure decreased from 161 $\pm 12.33 \mathrm{mmHg}$ at baseline to $148 \pm 7.27 \mathrm{mmHg}$ four weeks $(P<0.05)$ and to $132 \pm 6.13 \mathrm{mmHg}$ three months after the operation $(P<0.01)$, the diastolic blood pressure decreased from $102 \pm$ $4.99 \mathrm{mmHg}$ to $95 \pm 4.61 \mathrm{mmHg}$ four weeks $(P<0.05)$ and to $81 \pm 4.26 \mathrm{mmHg}$ three months after the operation $(P<0.01)$. The IPSS reduced from $21 \pm 4.82$ to $15 \pm 3.66$ four weeks $(P<0.05)$ and to $12 \pm 3.11$ three months after the operation $(P<0.01)$. The $Q \max$ were improved from $7 \pm 4.41$ to $12 \pm 1.34$ four weeks and to $15 \pm 2.92$ three months after the operation $(P<0.05)$. There were no notable adverse events. Conclusions: PKRP combined with terazosin in treating BPH with concomitant hypertension patient is a safe and effective procedure with a good patient compliance.

\section{Keywords}

Benign Prostatic Hyperplasia, Hypertension, Terazosin, Plasma Kinetic Vaporization of the Prostate

Corresponding author. 


\section{Introduction}

Benign prostatic hyperplasia (BPH) is a common disease in aging men. Surgical treatment plays an important role in symptomatic patients. A variety of techniques, from open prostatectomy to minimally invasive procedures have been performed. Transurethral resection of the prostate (TURP) remains the "gold standard” therapy for $\mathrm{BPH}$ [1] [2]. However, there are many BPH patients who had hypertension either before or during the $\mathrm{BPH}$ attack, which increases the risk of surgical intervention [1] [3] [4]. Therefore, a more optimally surgical procedure and ideal medical therapy would be required.

Plasma kinetic vaporization of the prostate (PKRP), a newly type of TURP, is regarded as less bleeding and easy control with high safety procedure [5] [6]. Adrenergic receptor blocker-terazosin hydrochloride is widely used for BPH patient, especially those accompany with hypertension, since the drug possessed effect of relieving the prostatic smooth muscle spasm and lowering the blood pressure [7]. The present study was designed to explore the effectiveness and safety of PKRP combined with terazosin medication for BPH with concomitant hypertension patient by analyses of the changes in diastolic and systolic blood pressures, IPSS and Qmax. The medication safety was assessed by the occurrence of adverse events.

\section{Patients and Methods}

A total of 205 patients aged 57 - 87 (mean 72) were enrolled in this study in the urology department from 2008 to 2012. Patients were excluded from the study if they had a history of neurogenic bladder dysfunction, prostate cancer, urethral stricture, secondary hypertension, unstable angina, myocardial infarction, cerebrovascular disease, orthostatic hypotension, and undergone previous prostatic or urethral surgery. The inclusion criteria were that they were BPH patients with indications for surgical intervention based on the symptomatic evaluation and related examinations. They had hypertension with the systolic blood pressure $\geq 140 \mathrm{mmHg}$ or diastolic blood pressure $\geq 90 \mathrm{mmHg}$. They were willing to accept the PKRP procedure and terazosin medication. All the patients were signed informed consent that confirmed by the hospital's ethics committee.

The subjects underwent PKRP and accepted the oral-terazosin hydrochloride (Shanghai Pharmaceutical Company Limited, China) one week before and three months after the operation. The drug administration was 1 - $6 \mathrm{mg}$ per day depending on the levels of hypertension and drug tolerance. They were received the follow-up for 3 months after the operation.

Therapeutic effect was determined by the changes in systolic blood pressure, diastolic blood pressure, international prostate symptom score (IPSS) and maximum urinary flow rate (Qmax), which were measured 4 weeks and 3 months after the PKRP operation. The data were compared with the controls as measured before the terazosin medication. The therapeutic safety was determined by analysis of the incidence of adverse drug reaction, e.g. the incidences of dizziness, palpitations, nausea and others, including addicted saliva, blurred vision, headache, flatulence, flushing.

\section{Statistical Analysis}

Data were analyzed by using statistical software SPSS (version 17.0 for Windows, SPSS, Chicago, IL, USA). All data are presented as mean \pm SD. one-way ANOVA followed by Bonferroni test was used for comparisons. $P<0.05$ was considered as statistically significant.

\section{Results}

All the subjects fulfilled the investigation and received the follow-up for three months. The benefit effect of the therapeutic procedure was revealed in that the positive changes in the accessed parameters. All the changes are significantly different from the value of the controls (base line).

Figure 1 shows the blood pressure changes recorded 4 weeks and 3 months follow-up after the operation. Both systolic blood pressure (A) and diastolic blood pressure (B) were significantly decreased compared with the base line (before the operation). The systolic blood pressure 4 weeks after the operation was decreased from $161 \pm 12.33$ to $148 \pm 7.27 \mathrm{mmHg}(P<0.05)$, and to $132 \pm 6.13 \mathrm{mmHg}(P<0.01) 3$ months after the operation. The diastolic blood pressure 4 weeks after the operation was decreased from $102 \pm 4.99$ to $96 \pm 4.61 \mathrm{mmHg}(P$ $<0.05)$, and to $81 \pm 4.26 \mathrm{mmHg}(P<0.01) 3$ months after the operation.

Compare with the base line (before the operation), the IPSS score was significantly decreased 4 weeks and 3 
months after the operation (from $21 \pm 4.82$ to $15 \pm 3.66, P<0.05$ ) and (from $21 \pm 4.82$ to $12 \pm 3.11, P<0.01$ ), respectively (Figure 2).

The beneficial effects of PKRP combined with terazosin medication treatment were also demonstrated, manifesting on the improvement of Qmax obtained from urodynamic assessment. Compare with the base line, the Qmax (ml/s) was remarkable increased 4 weeks and 3 months after the operation (from $7.63 \pm 4.41$ to $12.55 \pm$ 1.34, $P<0.05$ ) and (from $7.63 \pm 4.41$ to $15.51 \pm 2.92, P<0.01$ ), respectively (Figure 3 ).

\section{Safety and Compliance Analysis}

The adverse events in the terazosin medication were reasonable low. Of all the cases, only 5.03\% had dizziness, 2.64\% had palpitations, $1.98 \%$ had nausea, $4.73 \%$ had addicted to spit and blurred vision, headache, flatulence, flushing and other symptoms. The drug compliance is good. After 3 months, there were still 110 patients (89\%) remained the terazosin medication for the hypertension control.

\section{Discussion}

Benign prostatic hyperplasia (BPH) and hypertension are common diseases in old people and the incidence continues to increase with age. According to the statistics, man in 45-year-old appeared prostatic hyperplasia syndrome accounts for $23 \%$, while in 60 - 85 years of age accounts for $78 \%$, hypertension in the age over 70 accounts for $50 \%$. Epidemiological data reported by Maruenda et al. showed that about $25 \%$ of the population over the age of 60 had BPH together with hypertension [1]. Studies also revealed that hypertension was one of the
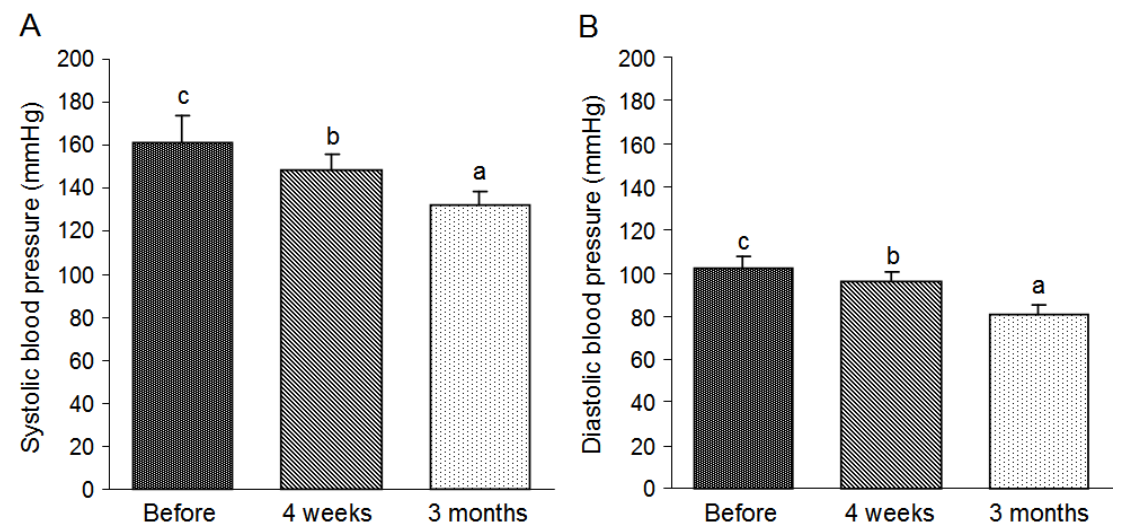

Figure 1. Changes in systolic blood pressure (A) and diastolic blood pressure (B) 4 weeks and 3 months after operation. Bars without a common superscript letter differ significantly (Mean $\pm \mathrm{SD}, \mathrm{n}=205, P<0.05$ ).

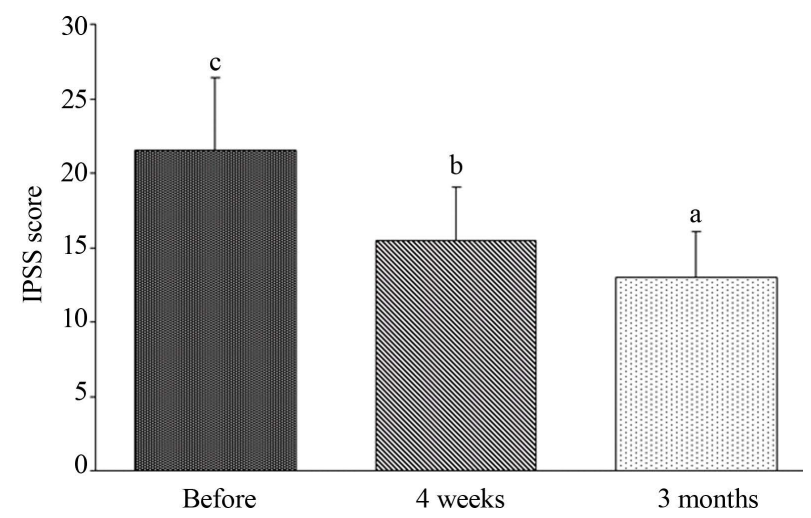

Figure 2. Changes in IPSS score 4 weeks and 3 months after operation. Bars without a common superscript letter differ significantly (Mean \pm SD, $\mathrm{n}=205, P<0.05$ ). 


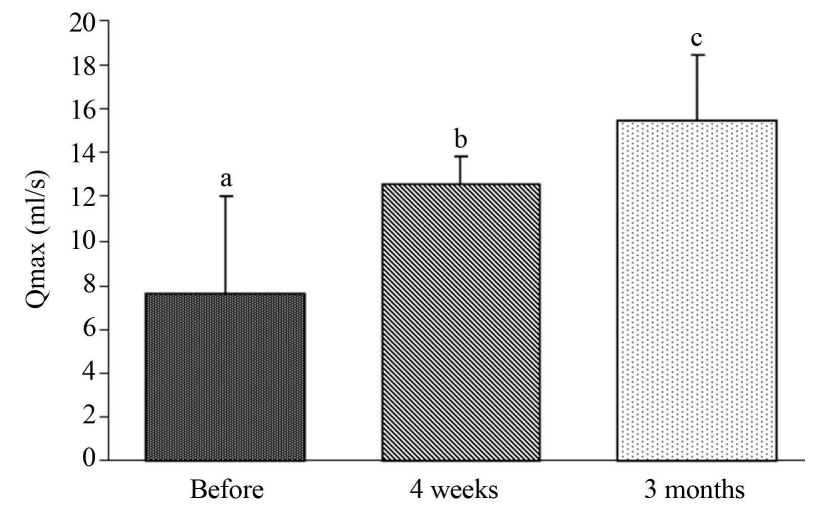

Figure 3. Changes in Qmax 4 weeks and 3 months after operation. Bars without a common superscript letter differ significantly (Mean $\pm \mathrm{SD}, \mathrm{n}=205, P<0.05)$.

independent risk factors for occurrence and progress of BPH [2]. Long-term hypertension, especially high diastolic blood pressure can promote the occurrence of BPH [3]. The investigation of Michel MC found that the BPH patient had the diastolic blood pressure $>90 \mathrm{mmHg}$ or had a history of hypertension, the IPSS was higher and the Qmax was lower significantly compared with those of normal blood pressure [4]. In addition, the BPH symptoms would get worse when more number of antihypertensive drugs is required.

The severity of hypertension is closely related to the increase in expression of vascular endothelial growth factor (VEGF) in prostatic stroma. The high expression of VEGF indicates the abnormal angiogenesis, progressive BPH and hypertension. The study of Guo Lijun et al. revealed that in BPH patient with coexisting hypertension, the prostate volume was much larger, the prostate stromal expression of microvessel density (MVD) and VEGF was remarkably higher, and the incidence of hematuria was obviously increased compared with the BPH patients without coexisting hypertension [3] [8].

Usually, the hypertension received the diagnosis and treatment ahead of the BPH. In such patients, when drug therapy for BPH interfered with the scheme of hypertension medication, severe consequences such as rebound in blood pressure and heart failure might happen [9]. Therefore, it is crucial to find out a proper treatment schedule, which should take into account both BPH and hypertension problems. The protocol of PKRP surgery combine with terazosin medication as we carried out in the present study revealed to be an ideal strategy.

The common pathogeneses of BPH and hypertension are sympathetic excitation [10]. Therefore, treatment for sympathetic disorders would benefit these two diseases. Terazosin, a long-acting selective $\alpha 1$-blockers, is widely used to the patient with hypertension and symptomatic BPH. By acting on the postganglionic $\alpha 1$ adrenergic receptors, the peripheral vessels will be dilated and the vascular resistance reduced. Thus, the blood pressure will be decreased, including both systolic blood pressure and diastolic blood pressure, while by acting on smooth muscle $\alpha 1$ adrenergic receptors in the bladder neck and prostate, the smooth muscle of bladder neck, prostate and prostatic capsule will be relaxed without affecting the detrusor function. Thus the resistance of bladder outlet and urethra will be reduced and the obstructive symptoms resulted from BPH will be relived. Recently, the safety and efficacy of terazosin have been proved in BPH patient of Asian population [11].

Roehrborn et al. conducted a randomized, double-blind, placebo-controlled study in 15 university medical centers and 141 community private urology clinic in the United States. A total of 2084 BPH cases were assigned to the investigation for 12-month. The patients were divided into terazosin group and placebo group. During the treatment, when the medication efficiency is less than 35\%, the dosage was then increased by increments from 2 $\mathrm{mg}$ to $10 \mathrm{mg} /$ day. By this way, the AUA symptom score was reached to $38 \%$ and the QOL was raised to $33 \%$ in terazosin treatment group, much higher than those in placebo group [12]. A multi-center, prospective clinical study on BPH patients by Du et al., found that the IPSS all decreased after 4 weeks terazosin medication. There were no clear-cut effect differences in different age and different prostate volumes, no matter $5 \alpha$-reductase inhibitors were applied [13]. In consistent, excellent efficacy, reliable safety and satisfied patient compliance of terazosin in treatment of BPH accompany with hypertension patients were demonstrated in the present study.

Imbalance of prostatic cell proliferation and apoptosis are the other important factors in causing BPH. Turkeri et al. detected that after 4 weeks treatment of $\alpha 1$-adenosine receptor ( $\alpha 1$-AR) blockers in BPH patients, the se- 
rum prostate specific antigen (PSA) was decreased 14\%, and the prostate apoptosis rate was much higher than those without $\alpha 1$-AR blockers medication [14]. Related studies on BPH patients exhibited that $\alpha 1$-AR blockers (terazosin) combined with 5a-reductase inhibitor (finasteride) had much better effect than single use of terazosin or finasteride, either on increase in prostate apoptosis rate or on relief of clinical symptoms, suggesting that a1-AR plays an important role in prostate cell apoptosis [15] [16].

In this study, the plasma ablation of the prostate was applied, which is not the heating process, the temperature of target tissue surface was only $40^{\circ} \mathrm{C}-70^{\circ} \mathrm{C}$ during the operation, and the heat penetration is not deep. The efficacy of plasma cutting is related to the tissue impedance. Since impedance of prostatic tissue is very high and prostatic capsule is lower, the cutting efficacy for prostatic tissue will be much higher than for prostatic capsule. Therefore, the probability of cutting through the prostatic capsule is very low and the damage of erectile nerve and vascular bundle outside the capsular could be effectively avoided.

A large number of studies showed that PKRP is an ideal procedure for the symptomatic BPH patient, in that the Qmax, residual urine volume (PVR), IPSS and QOL of BPH patients were remarkably improved after the surgery [6] [17] [18]. Compared with traditional TURP, our experience revealed that PKRP has several advantages, e.g. less bleeding, shorter operative time and relatively less complications either in perioperative stage or postoperative stage. During the operation, we paid great attention to avoid too long time of coagulation or electrical excision near the area of prostate capsule (especially 5,7 points) to minimize thermal tissue damage. By this way, the incidences of prostate capsule perforation and damage of neurovascular bundle on the surface of capsular were efficiently eliminated and the erectile function was greatly protected. The operative time was 25 $90 \mathrm{~min}$ and bleeding about $30-150 \mathrm{ml}$ for each patient in this study.

We believe that the PKRP surgery assisted with terazosin medication for treatment of BPH with concomitant hypertension patients is an excellent strategy. With this protocol, the advantages of both medication and surgical procedure were optimized so that the efficient and safety of the therapy could be obtained as we showed here. Among the side effects of $\alpha$-blockers as terazosin hydrochloride, dizziness is the most common adverse event, some patients had orthostatic hypotension, which influence the medication compliance. Nevertheless, it was found that dizziness and neurasthenia were independent of changes in blood pressure, suggesting other pathogenesis mechanisms might be involved, e.g. blockage of the adrenergic receptors in the central nervous system [19]. Yang et al. reported that the patients who underwent BPH surgery and received terazosin medication perioperatively presented an effectively shorten in postoperative indwelling catheter, more quickly alleviated the lower urinary tract symptoms (LUTS), and no detectable cardiovascular side effects [20]. Our patients were all informed about the possible side effect of terazosin before the medication. During the medication, they received closely observation and carefully dosage adjustment according to the therapy effects and drug reactions. No obvious adverse reaction associated with terazosin was noticed and the patient compliance was rather good. Three months follow-up after the operation, all the patients kept up the terazosin medication for normalized the blood pressure without any problem.

\section{Conclusion}

The beneficial effects of PKRP combined terazosin for treatment of BPH patient with coexisting hypertension were demonstrated in this study. The results of four weeks and three months follow-up after the operation showed that good control of blood pressure, quick alleviation of LUTS, reduction of IPSS and improvement of Qmax were all pronounced. Based on the satisfied surgical efficacy, reliable drug safety and good patient compliance, the procedures are highly recommended in treatment of symptomatic BPH with hypertension patient.

\section{Declaration of Interest}

The authors report no conflicts of interest. The authors alone are responsible for the content and writing of the paper.

\section{Acknowledgements}

This study was supported by the Qingyuan City People’s Hospital of Jinan University, China.

\section{References}

[1] Maruenda, J., Bhatnagar, V. and Lowenthal, D.T. (1999) Hypertension in the Elderly with Coexisting Benign Prostatic 
Hyperplasia. Urology, 53, 7-12. http://dx.doi.org/10.1016/S0090-4295(98)00533-0

[2] Pinheiro, L.C. and Martins Pisco, J. (2012) Treatment of Benign Prostatic Hyperplasia. Techniques in Vascular and Interventional Radiology, 15, 256-260. http://dx.doi.org/10.1053/j.tvir.2012.09.004

[3] Guo, L., Zhang, X., Li, P. and Na, Y. (2005) Association Study between Benign Prostatic Hyperplasia and Primary Hypertension. Zhonghua Wai Ke Za Zhi, 43, 108-111.

[4] Michel, M.C., Heemann, U. and Schumacher, H. (2004) Association of Hypertension with Symptoms of Benign Prostatic Hyperplasia. Journal of Urology, 172, 1390-1393. http://dx.doi.org/10.1097/01.ju.0000139995.85780.d8

[5] Nuhoğlu, B., Ayyildiz, A., Karagüzel, E. and Germiyanoğlu, C. (2006) Plasmakinetic Prostate Resection in the Treatment of Benign Prostate Hyperplasia: Results of 1-Year Follow-Up. International Journal of Urology, 13, 21-24. http://dx.doi.org/10.1111/j.1442-2042.2006.01218.x

[6] Zhao, X., Sun, X., Liu, Z., Wang, J. and Liu, L. (2006) Comparison of the Results of Transurethral Plasmakinetic Resection versus Transurethral Resection of the Prostate for Benign Prostatic Hyperplasia. Chinese Journal of Urology, 27, 628-630.

[7] Kaplan, S.A. and Kaplan, N.M. (1996) Alpha-Blockade: Monotherapy for Hypertension and Benign Prostatic Hyperplasia. Urology, 48, 541-550. http://dx.doi.org/10.1016/S0090-4295(96)00242-7

[8] Pareek, G., Shevchuk, M., Armenakas, N.A., Vasjovic, L., Hochberg, D.A., Basillote, J.B., et al. (2003) The Effect of Finasteride on the Expression of Vascular Endothelial Growth Factor and Microvessel Density: A Possible Mechanism for Decreased Prostatic Bleeding in Treated Patients. Journal of Urology, 169, 202-231. http://dx.doi.org/10.1016/S0022-5347(05)64025-6

[9] Walma, E.P. (1997) Withdrawal of Long Term Diuretic Medication in Elderly Patients: A Double Blind Randomized Trial. British Medical Journal, 315, 464-468. http://dx.doi.org/10.1136/bmj.315.7106.464

[10] Kobayashi, S., Tang, R. and Shapiro, E. (1993) Characterization Localization of Prostatic Alpha l Aderenoceptors Using Radio Ligand Binding on Slide-Mounted Tissue Section. Journal of Urology, 150, 2002-2006.

[11] Kwak, C., Lee, J.K. and Ku, J.H. (2007) High-Dose Terazosin Therapy (5 mg) in Korean Patients with Lower Urinary tract Symptoms with or Without Concomitant Hypertension: A Prospective, Open-Label Study. Yonsei Medical Journal, 48, 994-1000. http://dx.doi.org/10.3349/ymj.2007.48.6.994

[12] Roehrborn, C.G., Oesterling, J.E., Auerbach, S., Kaplan, S.A., Lloyd, L.K., Milam, D.E., et al. (1996) The Hytrin Community Assessmeng Trial Study: A One-Year Study of Terazosin versus Placebo in the Treatment of Men with Symptomatic Benign Prostactic Hyperplasia. Urology, 47, 159-168. http://dx.doi.org/10.1016/S0090-4295(99)80409-9

[13] Du, G., Qi, J., Song, J., Wang, Q., Kong, X., Jin, C., et al. (2010) A Multicenter Prospective Clinical Study on the Effectiveness and Safety of Terazosin in the Treatment of Chinese Benign Prostatic Hyperplasia Patients. Chinese Journal of Urology, 31, 343-346.

[14] Turkeri, L.N., Oxyurek, M., Ersev, D. and Akdaş, A. (2001) Apoptotic Regression of Prostatic Tissue Induced by Short-Term Doxazosin Treatment in Benign Prostatic Hyperplasia. Archivos Españoles de Urología, 54, 191-196.

[15] Glassman, D.T., Chon, J.K., Borkowski, A., Jacobs, S.C. and Kyprianou, N. (2001) Combined Effect of Terazosin and Finasteride on Apoptosis, Cell Proliferation, and Transforming Growth Factor-Beta Expression in Benign Prostatic Hyperplasia. The Prostate, 46, 45-51. http://dx.doi.org/10.1002/1097-0045(200101)46:1<45::AID-PROS1007>3.0.CO;2-U

[16] Savate, S.J., Spuregen, A.M., Galea, G., Britanico, J. and Vapnek, J.M. (1998) Combination Medical Therapy for Symptomatic Benign Prostatic Hyperplasia. The Canadian Journal of Urology, 5, 578-584.

[17] Autorino, R., Damiano, R., Di Lorenzo, G., Quarto, G., Perdonà, S., D’Armiento, M., et al. (2009) Four-Year Outcome of a Prospective Randomised Trial Comparing Bipolar Plasmakinetic and Monopolar Transurethral Resection of the Prostate. European Urology, 55, 922-929. http://dx.doi.org/10.1016/j.eururo.2009.01.028

[18] Ho, H.S., Yip, S.K., Lim, K.B., Fook, S., Foo, K.T. and Cheng, C.W. (2007) A Prospective Randomized Study Comparing Monopolar and Bipolar Transurethral Resection of Prostate Using Transurethral Resection in Saline (TURIS) System. European Urology, 52, 517-522. http://dx.doi.org/10.1016/j.eururo.2007.03.038

[19] Lepor, H., Jones, K. and Williford, W. (2001) The Mechanism of Adverse Events Associated with Terazosin: An Analysis of the Veterans Affairs Cooperative Study. Journal of Urology, 58, 508-516.

[20] Yang, C. and Yin, X. (2005) Observed Terazosin Efficacy in Perioperative Period of Transurethral Prostatectomy. Shandong Medical Journal, 45, 27. 